\title{
FACTORES ASOCIADOS A LA DESNUTRICIÓN CRÓNICA INFANTIL EN ECUADOR. ESTUDIO BASADO EN MODELOS DE REGRESIÓN Y ÁRBOLES DE CLASIFICACIÓN.
}

\section{Factors associated with chronic child malnutrition in Ecuador. A study based on regression models and classification trees.}

${ }^{1}$ Pablo Javier Flores Muñoz* (iD, ${ }^{2}$ Giorgia Nohelia Congacha Ortega (iD).

${ }^{1}$ Escuela Superior Politécnica de Chimborazo, Facultad de Ciencias, Grupo de Investigación en Ciencia de Datos, Riobamba, Ecuador.

${ }^{2}$ BASICALATAM SA, Human Data Department. Antonio de Ulloa N34-112, Quito, Ecuador.

*p_flores@espoch.edu.ec

$\mathrm{R}$ esumen

La presente investigación busca determinar los factores que influyen significativamente en la desnutrición crónica en niños de 0 a 5 años de edad en el Ecuador. Las variables que formaron parte del estudio han sido consideradas de acuerdo al marco conceptual propuesto por la UNICEF y extraídas de las bases de datos de la última encuesta de Salud y Nutrición 2018 desarrollada por el Instituto Nacional de Estadística y Censos y el Ministerio de Salud Pública. Con el objetivo de comparar resultados se aplicaron modelos basados en árboles de clasificación y de regresión logística. Se encontró que los factores básicos relacionados con: el grupo étnico del niño, la escolaridad de la madre, el acceso a comunicación móvil, el estado civil de los padres, la edad de la madre, el número de hijos en el hogar y el tipo de combustible que se usa para cocinar influyen de manera significativa sobre el estado nutricional del infante. Estos factores están relacionados directamente con factores básicos y subyacentes como control de la madre antes y después del parto, la vacunación del infante, la adecuada alimentación y el tamaño del niño al nacer, los cuales también influyen en la desnutrición.

Palabras claves: Desnutrición infantil, Regresión, Árboles de decisión.

\section{A bstract}

This research seeks to determine significantly influencing factors on chronic malnutrition of children from zero to five years old in Ecuador. The variables that were part of the study have been considered according to the conceptual framework proposed by UNICEF and extracted from the databases of the latest 2018 Health and Nutrition survey developed by the National Institute of Statistics and Censuses and the Ministry of Public Health. In order to compare results, models based on classification trees and logistic regression were applied. It was found that the basic factors related to: child's ethnic group, mother's schooling, access to mobile communication, parents' marital status, mother's age, the number of children in the family, and the type of fuel used for cooking have a significant influence on the infant's nutritional status. These factors are directly related with basic and underlying factors such as control of the mother before and after delivery, infant vaccination, adequate nutrition and the size of the child at birth, which also influence in malnutrition.

Keywords: Child malnutrition, ENSANUT, Regression, Decision trees. 


\section{INTRODUCCIÓN}

La desnutrición crónica infantil, principalmente en niños menores de 5 años, se asocia directamente a problemas de crecimiento, desarrollo físico, cognitivo y emocional, lo cual tiene un impacto directo en el rendimiento escolar y posterior capacidad de trabajo que tenemos los seres humanos a lo largo de la vida (1). Es así que este hecho constituye no solo un problema de salud pública, sino además un problema social que afecta directamente a una nación, la cual tiene el deber de hacer los esfuerzos necesarios para erradicarla. En el Ecuador, las cifras publicadas por el Instituto Nacional de Estadísticas y Censos (INEC), revelan, de acuerdo con el último estudio, que la prevalencia de desnutrición crónica en menores de cinco años es del $23.0 \%$, la cual no presenta una diferencia estadísticamente significativa con el anterior periodo analizado (2). Aunque lo ideal sería erradicar este problema, al parecer, queda mucho trabajo pendiente para alcanzar al menos la meta de nuestro actual plan nacional de desarrollo, de reducir esta prevalencia a $13.2 \%(3)$.

La Encuesta Nacional de Salud y Nutrición ENSANUT 2018 es una operación estadística realizada por el INEC y el Ministerio de Salud Pública del Ecuador (MSP), cuyo objetivo principal es "generar indicadores sobre los principales problemas y la situación de salud de la población ecuatoriana con el fin de evaluar y generar las políticas públicas en temas de salud y nutrición" (4), para ello, recolecta la información en 5 formularios, que sirven para generar 9 bases de datos diferentes.

El proceso de planificación, diseño, construcción, recolección, capacitación, procesamiento, análisis, difusión y evaluación de esta encuesta se la completó en un periodo de 965 días. Sin duda, la información obtenida es muy extensa y valiosa, ya que basa su metodología en procedimientos de alta calidad establecidos por la Organización Mundial de la Salud (OMS) y la UNICEF. En el año 2020, se publicaron los resultados de esta encuesta en la página web oficial del INEC, donde principalmente se puede observar indicadores (tasas, porcentajes, promedios) de las variables estudiadas, y en particular de la variable de interés (desnutrición crónica). Aunque esta descripción, nos permite identificar la situación actual y retrospectiva de la desnutrición en nuestro país, creemos que los esfuerzos deben estar orientados a análisis más complejos, que nos permitan conocer las principales causas por las que se origina la desnutrición, lo cual, se podría constituir en un referente para la toma de decisiones, que permita destinar recursos y aplicar políticas adecuadas, enfocadas directamente a los factores influyentes y no a otros que posiblemente no generarían ningún efecto significativo sobre la problemática. Al respecto, poco o nada se ha hecho dentro de nuestra región y en particular de nuestro país. Hablando de Latinoamérica y el Caribe, donde vivimos una realidad similar, son muy pocos los países que han invertido esfuerzos en investigar estas causas.

Tradicionalmente, los modelos matemáticos de regresión se han utilizado para determinar la influencia significativa de variables (o factores) independientes sobre una dependiente, como por ejemplo el caso de la desnutrición crónica infantil (5) que es el objeto del presente estudio, sin embargo, el problema del cumplimiento previo de supuestos asociados a estos modelos, así como el advenimiento de nuevas técnicas algorítmicas de clasificación, han hecho que se puedan comparar la efectividad de estos enfoques. No decimos que una metodología en general sea mejor que otra, pero sí que, de acuerdo con la naturaleza de los datos obtenidos, una podría arrojar mejores resultados. De hecho, se ha convertido ya en una tendencia, en varias áreas del conocimiento comparar estos enfoques, mediante modelos apropiados con el fin de encontrar uno que clasifique adecuadamente una variable en función de otras $(6,7,8,9)$.

La presente investigación, haciendo uso de la información recolectada en la última encuesta de salud y nutrición ENSANUT 2018, busca encontrar el mejor modelo, que permita determinar adecuadamente los principales factores significativos asociados a la desnutrición crónica, en niños de 0 a 5 años de edad, con el fin de contribuir en el proceso de toma de decisiones en políticas públicas eficientes, orientadas a mitigar esta pro- 
blemática. En el siguiente capítulo, se explica el marco conceptual de la UNICEF que permite justificar la elección de posibles factores asociados a la desnutrición crónica y las variables medidas en la ENSANUT con las que se relacionan.

Además, se explica el proceso de operacionalización de las bases de datos y su posterior análisis. En el capítulo III se muestran los principales resultados al aplicar modelos matemáticos y algorítmicos sobre la base de datos operacionalizada. Posteriormente, en el capítulo IV, se realiza una discusión sobre el poder predictivo y eficiencia de ambas metodologías y finalmente en el Capítulo $\mathrm{V}$, se concluye sobre el peso que tienen los factores más influyentes analizados sobre la desnutrición crónica infantil.

\section{MATERIALES Y MÉTODOS}

\section{MARCO CONCEPTUAL DE LA UNICEF PARA ANALIZAR FACTORES ASOCIADOS A LA DESNUTRICIÓN.}

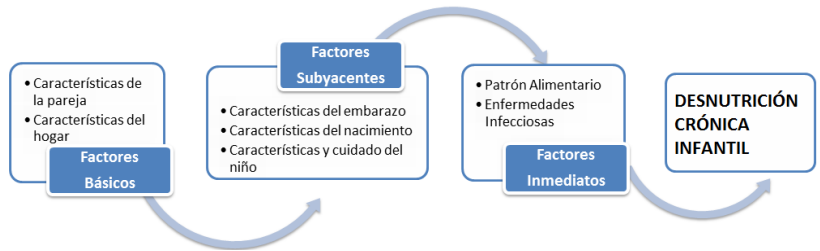

Figura 1. Posibles factores asociados a la desnutrición crónica, de acuerdo al marco conceptual propuesto por la UNICEF

Fuente: Elaboración Propia.

En la década de los noventa, el Fondo de las Naciones Unidas para la Infancia (UNICEF) propuso un marco conceptual para analizar los factores asociados a la desnutrición infantil, el cual considera que dichos factores pueden clasificarse en tres grupos: Inmediatos, Subyacentes y Básicos. Los factores inmediatos estarían asociados directamente a la desnutrición crónica infantil, mismos que a su vez son intermediadores de una asociación indirecta que existe entre los factores básicos y subyacentes (10).

En este contexto, aunque en principio los factores básicos podrían presentar una significativa asociación con la desnutrición crónica infantil, estos al estar intermediados por factores subyacentes e inmediatos crean una relación que tiende a dis- minuir la significativa asociación inicial, es decir, se crea una mayor precisión en la medición de la asociación entre los factores básicos y la variable de interés.

Del mismo modo, los factores subyacentes, aunque inicialmente podrían presentar una significativa asociación con la desnutrición, estos al estar intermediados por factores inmediatos, y a su vez condicionados por la relación con los factores básicos, crean una relación que tiende a disminuir la significativa asociación inicial, con lo que de igual manera se consigue una mayor precisión en la medición de la asociación entre los factores subyacentes y la desnutrición infantil.

\begin{tabular}{|c|c|c|}
\hline Factores & Código & Variable / item \\
\hline \multirow{4}{*}{$\begin{array}{l}\text { Factores } \\
\text { Básicos }\end{array}$} & FBCP1_1 & Años cumplidos de la madre \\
\hline & FBCP1_5 & Número de hijos nacidos vivos \\
\hline & FBCH_11 & Número de cuartos de la vivienda \\
\hline & FBCH_12 & Número de dormitorios de la vivienda \\
\hline \multirow[t]{22}{*}{$\begin{array}{l}\text { Factores } \\
\text { Subyacentes }\end{array}$} & FSCE_3 & $\begin{array}{l}\text { Meses de embarazo cuando se hizo el } \\
\text { primer control }\end{array}$ \\
\hline & FSCE_4 & Cuántos controles tuvo antes del parto \\
\hline & FSCE_11 & Primer control postparto-días \\
\hline & FSCE_12 & Primer control postparto-semanas \\
\hline & FSCE_13 & Primer control postparto-meses \\
\hline & FSCN_10 & Talla al nacer \\
\hline & FSCCN_2 & control por primera vez-días \\
\hline & FSCCN_3 & control por primera vez-semanas \\
\hline & FSCCN_4 & control por primera vez-meses \\
\hline & FSCCN_8 & bcg-dosisf41002a \\
\hline & FSCCN_9 & hepatitis b-dosis \\
\hline & FSCCN_10 & pentavalente 1 -dosis \\
\hline & FSCCN_11 & pentavalente 2 -dosis \\
\hline & FSCCN_12 & pentavalente 3 -dosis \\
\hline & FSCCN_13 & rotavirus 1 -dosis \\
\hline & FSCCN_14 & rotavirus 2-dosis \\
\hline & FSCCN_15 & antipolio(opv) 1-dosis \\
\hline & FSCCN_16 & antipolio(opv) 2-dosis \\
\hline & FSCCN_17 & antipolio(opv) 3-dosis \\
\hline & FSCCN_18 & neumococo 1 -dosis \\
\hline & FSCCN_19 & neumococo 2-dosis \\
\hline & FSCCN_22 & Edad (en meses) \\
\hline \multirow{5}{*}{$\begin{array}{l}\text { Factores } \\
\text { Inmediatos }\end{array}$} & FIPA_1 & Hasta qué edad le dio el seno-días \\
\hline & FIPA_2 & Hasta qué edad le dio el seno-meses \\
\hline & FIPA_3 & Hasta qué edad le dio el seno-años \\
\hline & FIPA_14 & $\begin{array}{l}\text { Consumió ayer -agua pura - cuántas } \\
\text { veces }\end{array}$ \\
\hline & FIPA_21 & Consumió ayer -sopa- cuántas veces \\
\hline
\end{tabular}

Tabla 1. Variables cuantitativas de la ENSANUT que contienen las características de los factores propuestos por el marco conceptual de la UNICEF Fuente: Elaboración Propia. 


\begin{tabular}{|c|c|c|c|}
\hline Factores & Código & Variable / item & $\begin{array}{l}\text { Categoría / Posible } \\
\text { respuesta }\end{array}$ \\
\hline $\begin{array}{l}\text { Variable } \\
\text { dependiente }\end{array}$ & & $\begin{array}{l}\text { Desnutrición } \\
\text { crónica menores } \\
\text { de } 5 \text { años }\end{array}$ & $\begin{array}{l}0: \mathrm{Si} \\
1: \mathrm{No}\end{array}$ \\
\hline \multirow[t]{7}{*}{$\begin{array}{l}\text { FACTORES } \\
\text { BÁSICOS }\end{array}$} & FBCP1_4 & $\begin{array}{l}\text { El padre vive } \\
\text { con usted en el } \\
\text { hogar }\end{array}$ & $\begin{array}{l}\mathrm{Si} \\
\text { No }\end{array}$ \\
\hline & FBCP1_6 & Grupo étnico & $\begin{array}{l}\text { Mestizo/a } \\
\text { Indígena } \\
\text { Montuvio/a } \\
\text { Blanco/a } \\
\text { Afroecuatoriano/a } \\
\text { Negro/a } \\
\text { Otro }\end{array}$ \\
\hline & FBCP1_7 & Estado civil & $\begin{array}{l}\text { Casado } \\
\text { Divorciado } \\
\text { Separado } \\
\text { Soltero } \\
\text { Unión de hecho } \\
\text { Unión libre } \\
\text { Viudo }\end{array}$ \\
\hline & FBCP1_8 & $\begin{array}{l}\text { Escolaridad de la } \\
\text { madre }\end{array}$ & $\begin{array}{l}\text { Ninguno o Centro de } \\
\text { Alfabetización } \\
\text { Educación Básica } \\
\text { Educación Media/ } \\
\text { Bachillerato } \\
\text { Superior }\end{array}$ \\
\hline & FBCH_1 & $\begin{array}{l}\text { Vía de acceso } \\
\text { principal a la } \\
\text { vivienda }\end{array}$ & $\begin{array}{l}\text { Calle pavimentada o } \\
\text { adoquinada } \\
\text { Empedrado } \\
\text { Lastrado/calle de tierra } \\
\text { otro } \\
\text { Río/mar } \\
\text { Sendero }\end{array}$ \\
\hline & FBCH_2 & Tipo de vivienda & $\begin{array}{l}\text { Casa o villa } \\
\text { Departamento } \\
\text { Mediagua } \\
\text { Rancho } \\
\text { Cuartos inquilinato } \\
\text { Choza } \\
\text { Otro }\end{array}$ \\
\hline & FBCH_3 & $\begin{array}{l}\text { Material } \\
\text { predominante } \\
\text { del techo }\end{array}$ & $\begin{array}{l}\text { Asbesto (Eternit) } \\
\text { hormigón/losa/cemento } \\
\text { otro } \\
\text { palma/paja/hoja } \\
\text { teja } \\
\text { zinc }\end{array}$ \\
\hline
\end{tabular}

\begin{tabular}{|c|c|c|}
\hline FBCH_4 & $\begin{array}{l}\text { Material } \\
\text { predominante de } \\
\text { las paredes }\end{array}$ & $\begin{array}{l}\text { adobe/tapia } \\
\text { asbesto/cemento } \\
\text { bahareque (caña, carrizo } \\
\text { revestido) } \\
\text { caña o estera } \\
\text { hormigón/bloque/ } \\
\text { ladrillo } \\
\text { madera } \\
\text { otra }\end{array}$ \\
\hline FBCH_5 & $\begin{array}{l}\text { Material } \\
\text { predominante } \\
\text { del piso }\end{array}$ & $\begin{array}{l}\text { cemento/ladrillo } \\
\text { cerámica/baldosa/vinyl } \\
\text { tabla/tablón no tratado } \\
\text { duela/parquet/ } \\
\text { tabloncillo/piso flotante } \\
\text { tierra } \\
\text { mármol/marmetón } \\
\text { otro }\end{array}$ \\
\hline FBCH_6 & $\begin{array}{l}\text { De donde } \\
\text { obtienen el agua }\end{array}$ & $\begin{array}{l}\text { carro repartidor/triciclo } \\
\text { otra fuente por tubería } \\
\text { otro } \\
\text { pila o llave pública } \\
\text { pozo } \\
\text { red pública } \\
\text { río/vertiente/acequia }\end{array}$ \\
\hline FBCH_7 & $\begin{array}{l}\text { El agua que } \\
\text { recibe la } \\
\text { vivienda es }\end{array}$ & $\begin{array}{l}\text { No recibe agua por } \\
\text { tubería } \\
\text { recibe por tubería } \\
\text { dentro de la vivienda } \\
\text { recibe por tubería fuera } \\
\text { de la vivienda pero } \\
\text { dentro del edificio } \\
\text { recibe por tubería fuera } \\
\text { del edificio }\end{array}$ \\
\hline FBCH_8 & $\begin{array}{l}\text { El servicio } \\
\text { higiénico de la } \\
\text { vivienda es: }\end{array}$ & $\begin{array}{l}\text { excusado y } \\
\text { alcantarillado } \\
\text { excusado y pozo ciego } \\
\text { excusado y pozo séptico } \\
\text { letrina } \\
\text { no tiene }\end{array}$ \\
\hline FBCH_9 & $\begin{array}{l}\text { El servicio de luz } \\
\text { eléctrica es: }\end{array}$ & $\begin{array}{l}\text { empresa eléctrica } \\
\text { pública } \\
\text { Ninguno } \\
\text { planta eléctrica privada } \\
\text { vela/candil/mechero/gas }\end{array}$ \\
\hline
\end{tabular}




\begin{tabular}{|c|c|c|c|}
\hline & FBCH_10 & $\begin{array}{l}\text { Principalmente } \\
\text { cómo eliminan } \\
\text { la basura: }\end{array}$ & $\begin{array}{l}\text { botan a la calle/ } \\
\text { quebrada/río } \\
\text { contratan el servicio } \\
\text { la entierran } \\
\text { la queman } \\
\text { otra } \\
\text { servicio municipal }\end{array}$ \\
\hline & FBCH_13 & $\begin{array}{l}\text { El agua que } \\
\text { toman los } \\
\text { miembros del } \\
\text { hogar: }\end{array}$ & $\begin{array}{l}\text { la beben tal como llega } \\
\text { al hogar } \\
\text { la hierven } \\
\text { no sabe } \\
\text { otro tratamiento }\end{array}$ \\
\hline & FBCH_14 & $\begin{array}{l}\text { Combustible } \\
\text { que utilizan para } \\
\text { cocinar }\end{array}$ & $\begin{array}{l}\text { electricidad (inducción) } \\
\text { gas } \\
\text { leña/carbón } \\
\text { no cocina }\end{array}$ \\
\hline & FBCH_15 & $\begin{array}{l}\text { ¿El servicio } \\
\text { higiénico } \\
\text { del hogar es } \\
\text { exclusivo? }\end{array}$ & $\begin{array}{l}\mathrm{Si} \\
\text { No }\end{array}$ \\
\hline & FBCH_16 & $\begin{array}{l}\text { ¿Disponen } \\
\text { de servicio } \\
\text { telefónico } \\
\text { convencional? }\end{array}$ & $\begin{array}{l}\mathrm{Si} \\
\text { No }\end{array}$ \\
\hline & FBCH_17 & $\begin{array}{l}\text { ¿Algún miembro } \\
\text { del hogar tiene } \\
\text { telf. celular? }\end{array}$ & $\begin{array}{l}\mathrm{Si} \\
\text { No }\end{array}$ \\
\hline & FBCH_18 & $\begin{array}{l}\text { La vivienda que } \\
\text { ocupa el hogar } \\
\text { es: }\end{array}$ & $\begin{array}{l}\text { anticresis y arriendo } \\
\text { cedida } \\
\text { en arriendo } \\
\text { otra } \\
\text { propia y la está pagando } \\
\text { propia y totalmente } \\
\text { pagada } \\
\text { recibida por servicios }\end{array}$ \\
\hline \multirow[t]{3}{*}{$\begin{array}{l}\text { Factores } \\
\text { Subyacentes }\end{array}$} & FSCE_1 & $\begin{array}{l}\text { Tuvo algún } \\
\text { control prenatal }\end{array}$ & $\begin{array}{l}\mathrm{Si} \\
\mathrm{No}\end{array}$ \\
\hline & FSCE_2 & $\begin{array}{l}\text { Dónde se hizo } \\
\text { el control } \\
\text { con mayor } \\
\text { frecuencia }\end{array}$ & $\begin{array}{l}\text { establecimiento de salud } \\
\text { del MSP } \\
\text { clínica/consultorio } \\
\text { privado } \\
\text { en casa } \\
\text { hospital/dispensario } \\
\text { del IESS } \\
\text { junta de beneficencia } \\
\text { seguro social campesino } \\
\text { otro }\end{array}$ \\
\hline & FSCE_5 & $\begin{array}{l}\text { En el embarazo } \\
\text { le vacunaron } \\
\text { contra el tétanos }\end{array}$ & $\begin{array}{l}\text { Si } \\
\text { No } \\
\text { No sabe/no responde }\end{array}$ \\
\hline
\end{tabular}

\begin{tabular}{|c|c|c|}
\hline FSCE_6 & $\begin{array}{l}\text { En qué lugar } \\
\text { tuvo el parto }\end{array}$ & $\begin{array}{l}\text { establecimiento de salud } \\
\text { del MSP } \\
\text { clínica/consultorio } \\
\text { privado } \\
\text { en casa } \\
\text { hospital/dispensario } \\
\text { del IESS } \\
\text { junta de beneficencia } \\
\text { seguro social campesino } \\
\text { otro }\end{array}$ \\
\hline FSCE_7 & $\begin{array}{l}\text { Qué persona o } \\
\text { profesional le } \\
\text { atendió }\end{array}$ & $\begin{array}{l}\text { médico } \\
\text { obstetra } \\
\text { familiar } \\
\text { comadrona o partera } \\
\text { enfermera } \\
\text { auxiliar de enfermería } \\
\text { otra }\end{array}$ \\
\hline FSCE_8 & El parto fue: & $\begin{array}{l}\text { cesárea } \\
\text { normal }\end{array}$ \\
\hline FSCE_9 & $\begin{array}{l}\text { El nacimiento } \\
\text { fue a los } 9 \\
\text { meses o antes de } \\
\text { tiempo }\end{array}$ & $\begin{array}{l}\text { a tiempo } \\
\text { no sabe } \\
\text { pos maduro } \\
\text { prematuro }\end{array}$ \\
\hline FSCE_10 & $\begin{array}{l}\text { Tuvo algún } \\
\text { control después } \\
\text { del parto }\end{array}$ & $\begin{array}{l}\mathrm{Si} \\
\mathrm{No}\end{array}$ \\
\hline FSCE_14 & $\begin{array}{l}\text { Dónde tuvo } \\
\text { el control } \\
\text { postparto }\end{array}$ & $\begin{array}{l}\text { establecimiento de salud } \\
\text { del MSP } \\
\text { clínica/consultorio } \\
\text { privado } \\
\text { hospital/dispensario } \\
\text { del IESS } \\
\text { otro, ¿cuál? } \\
\text { seguro social campesino } \\
\text { hospital ff.aa /policía } \\
\text { otro }\end{array}$ \\
\hline FSCN_1 & $\begin{array}{l}\text { Le pesaron en } \\
\text { el momento de } \\
\text { nacer }\end{array}$ & $\begin{array}{l}\mathrm{Si} \\
\mathrm{No}\end{array}$ \\
\hline FSCN_2 & $\begin{array}{l}\text { En que unidad } \\
\text { de medida fue } \\
\text { pesado }\end{array}$ & $\begin{array}{l}\text { gramos } \\
\text { kilogramos } \\
\text { libras-onzas } \\
\text { no sabe }\end{array}$ \\
\hline FSCN_5 & $\begin{array}{l}\text { Con respecto a } \\
\text { otros bebes el } \\
\text { tamaño de su } \\
\text { hijo era: }\end{array}$ & $\begin{array}{l}\text { igual } \\
\text { no sabe } \\
\text { pequeño } \\
\text { muy pequeño } \\
\text { más grande }\end{array}$ \\
\hline FSCN_6 & $\begin{array}{l}\text { Tiene el carnet } \\
\text { de salud infantil }\end{array}$ & $\begin{array}{l}\text { Si } \\
\text { No } \\
\text { Si le entregaron, pero se } \\
\text { perdió }\end{array}$ \\
\hline
\end{tabular}




\begin{tabular}{|c|c|c|c|}
\hline & FSCN_7 & $\begin{array}{l}\text { Registró el peso } \\
\text { al nacer }\end{array}$ & $\begin{array}{l}\mathrm{Si} \\
\text { No }\end{array}$ \\
\hline & FSCN_9 & $\begin{array}{l}\text { Registró la talla } \\
\text { al nacer }\end{array}$ & $\begin{array}{l}\mathrm{Si} \\
\text { No }\end{array}$ \\
\hline & FSCN_11 & $\begin{array}{l}\text { Registró el } \\
\text { perímetro } \\
\text { cefálico al nacer }\end{array}$ & $\begin{array}{l}\mathrm{Si} \\
\mathrm{No}\end{array}$ \\
\hline & FSCN_13 & $\begin{array}{l}\text { El carnet } \\
\text { registra puntos } \\
\text { en la curva de } \\
\text { crecimiento }\end{array}$ & $\begin{array}{l}\mathrm{Si} \\
\text { No }\end{array}$ \\
\hline & FSCCN_1 & $\begin{array}{l}\text { Después que } \\
\text { nació le llevó a } \\
\text { control médico }\end{array}$ & $\begin{array}{l}\mathrm{Si} \\
\text { No }\end{array}$ \\
\hline & FSCCN_5 & $\begin{array}{l}\text { Porqué o para } \\
\text { que lo llevó }\end{array}$ & $\begin{array}{l}\text { estaba enfermo } \\
\text { para control niño sano } \\
\text { no recuerda }\end{array}$ \\
\hline & FSCCN_6 & $\begin{array}{l}\text { A qué } \\
\text { establecimiento } \\
\text { de salud lo llevó }\end{array}$ & $\begin{array}{l}\text { establecimiento de salud } \\
\text { del MSP } \\
\text { clínica/consultorio } \\
\text { privado } \\
\text { hospital/dispensario } \\
\text { del IESS } \\
\text { seguro social campesino } \\
\text { otro } \\
\text { unidad municipal de } \\
\text { salud } \\
\text { otro, ¿cuál? }\end{array}$ \\
\hline & FSCCN_7 & $\begin{array}{l}\text { Vive con usted } \\
\text { actualmente }\end{array}$ & $\begin{array}{l}\mathrm{Si} \\
\text { No }\end{array}$ \\
\hline & FSCCN_21 & Grupo de edad & \begin{tabular}{|l}
$0-11$ \\
$12-18$ \\
$19-23$ \\
$24-30$ \\
$31-35$ \\
$48-59$ \\
otro
\end{tabular} \\
\hline & FSCCN_23 & Sexo del niño & $\begin{array}{l}\text { Hombre } \\
\text { Mujer }\end{array}$ \\
\hline $\begin{array}{l}\text { Factores } \\
\text { Inmediatos }\end{array}$ & FIPA_4 & $\begin{array}{l}\text { Fue alimentado } \\
\text { con leche } \\
\text { materna el día } \\
\text { de ayer }\end{array}$ & $\begin{array}{l}\mathrm{Si} \\
\text { No }\end{array}$ \\
\hline & FIPA_5 & $\begin{array}{l}\text { A qué tiempo } \\
\text { después del } \\
\text { nacimiento } \\
\text { empezó a mamar } \\
\text { o lactar }\end{array}$ & $\begin{array}{l}\text { entre una hora y menos } \\
\text { de } 24 \text { horas } \\
\text { después del parto } \\
\text { más de un día } \\
\text { menos de una hora }\end{array}$ \\
\hline
\end{tabular}

\begin{tabular}{|c|c|c|}
\hline FIPA_6 & $\begin{array}{l}\text { Los tres } \\
\text { primeros días } \\
\text { después del } \\
\text { nacimiento le } \\
\text { dió algo de beber } \\
\text { aparte de leche } \\
\text { materna }\end{array}$ & $\begin{array}{l}\mathrm{Si} \\
\text { No }\end{array}$ \\
\hline FIPA_11 & $\begin{array}{l}\text { Le dio pecho } \\
\text { cada vez que le } \\
\text { pidió }\end{array}$ & $\begin{array}{l}\mathrm{Si} \\
\text { No }\end{array}$ \\
\hline FIPA_12 & $\begin{array}{l}\text { Consumió algún } \\
\text { líquido diferente } \\
\text { a la leche } \\
\text { materna ayer }\end{array}$ & $\begin{array}{l}\mathrm{Si} \\
\text { No }\end{array}$ \\
\hline FIPA_13 & $\begin{array}{l}\text { Consumió ayer- } \\
\text { agua pura }\end{array}$ & $\begin{array}{l}\mathrm{Si} \\
\text { No } \\
\text { No sabe/no responde }\end{array}$ \\
\hline FIPA_15 & $\begin{array}{l}\text { Consumió ayer- } \\
\text { leche de fórmula }\end{array}$ & $\begin{array}{l}\mathrm{Si} \\
\text { No } \\
\text { No sabe/no responde }\end{array}$ \\
\hline FIPA_17 & $\begin{array}{l}\text { Consumió ayer- } \\
\text { leche en polvo }\end{array}$ & $\begin{array}{l}\mathrm{Si} \\
\text { No } \\
\text { No sabe/no responde }\end{array}$ \\
\hline FIPA_19 & $\begin{array}{l}\text { Consumió ayer- } \\
\text { jugos naturales }\end{array}$ & $\begin{array}{l}\mathrm{Si} \\
\text { No } \\
\text { No sabe/no responde }\end{array}$ \\
\hline FIPA_20 & $\begin{array}{l}\text { Consumió ayer- } \\
\text { sopa }\end{array}$ & $\begin{array}{l}\mathrm{Si} \\
\text { No } \\
\text { No sabe/no responde }\end{array}$ \\
\hline FIPA_22 & $\begin{array}{l}\text { Consumió ayer- } \\
\text { yogurt }\end{array}$ & $\begin{array}{l}\mathrm{Si} \\
\text { No } \\
\text { No sabe/no responde }\end{array}$ \\
\hline FIPA_24 & $\begin{array}{l}\text { Consumió ayer- } \\
\text { colada }\end{array}$ & $\begin{array}{l}\mathrm{Si} \\
\text { No } \\
\text { No sabe/no responde }\end{array}$ \\
\hline FIPA_26 & $\begin{array}{l}\text { Consumió ayer- } \\
\text { gaseosa }\end{array}$ & $\begin{array}{l}\mathrm{Si} \\
\text { No } \\
\text { No sabe/no responde }\end{array}$ \\
\hline FIPA_28 & $\begin{array}{l}\text { Consumió ayer- } \\
\text { agua aromática }\end{array}$ & $\begin{array}{l}\mathrm{Si} \\
\text { No } \\
\text { No sabe/no responde }\end{array}$ \\
\hline FIPA_30 & $\begin{array}{l}\text { Consumió ayer- } \\
\text { cualquier otro } \\
\text { líquido }\end{array}$ & $\begin{array}{l}\mathrm{Si} \\
\text { No } \\
\text { No sabe/no responde }\end{array}$ \\
\hline FIPA_32 & $\begin{array}{l}\text { Comió algún } \\
\text { alimento ayer }\end{array}$ & $\begin{array}{l}\mathrm{Si} \\
\text { No }\end{array}$ \\
\hline FIPA_33 & $\begin{array}{l}\text { Comió colada } \\
\text { espesa de harina } \\
\text { de trigo }\end{array}$ & $\begin{array}{l}\mathrm{Si} \\
\text { No } \\
\text { No sabe/no responde }\end{array}$ \\
\hline
\end{tabular}




\begin{tabular}{|l|l|l|l|}
\hline \multirow{7}{*}{} & FIPA_34 & $\begin{array}{l}\text { Comió colada } \\
\text { espesa de granos }\end{array}$ & $\begin{array}{l}\text { Si } \\
\text { No } \\
\text { No sabe/no responde }\end{array}$ \\
\cline { 2 - 4 } & FIPA_35 & $\begin{array}{l}\text { Comió zapallo, } \\
\text { zanahoria, } \\
\text { pepino }\end{array}$ & $\begin{array}{l}\text { Si } \\
\text { No } \\
\text { No sabe/no responde }\end{array}$ \\
\cline { 2 - 4 } & FIPA_36 & $\begin{array}{l}\text { Comió papa } \\
\text { blanca, yuca, } \\
\text { camote }\end{array}$ & $\begin{array}{l}\text { Si } \\
\text { No } \\
\text { No sabe/no responde }\end{array}$ \\
\cline { 2 - 4 } & FIEI_1 & $\begin{array}{l}\text { Ha tenido } \\
\text { diarrea en las } \\
\text { últimas dos } \\
\text { semanas }\end{array}$ & $\begin{array}{l}\text { Si } \\
\text { No } \\
\text { No sabe/no responde }\end{array}$ \\
\cline { 2 - 4 } & $\begin{array}{l}\text { En las dos } \\
\text { últimas semanas } \\
\text { ha tenido tos o } \\
\text { moquera }\end{array}$ & $\begin{array}{l}\text { Si } \\
\text { No }\end{array}$ \\
\hline
\end{tabular}

Tabla 2. Variables cualitativas de la ENSANUT que contienen las características de los factores propuestos por el marco conceptual de la UNICEF. Fuente: Elaboración Propia.

En la Figura 1 se muestran las características que de acuerdo al marco conceptual en cuestión deben ser medidas en cada uno de estos factores, mismas que encuentran asociadas con la información recolectada en la ENSANUT 2018 de acuerdo al detalle presentado en la Tabla 1 para variables cuantitativas y Tabla 2 para cualitativas. Además, con el fin de que sirva de guía en el proceso de operacionalización de bases de datos, se muestran las categorías o posibles respuestas que tienen estas variables.

\section{PROCESO DE OPERACIONALIZACIÓN DE LAS BASES DE DATOS.}

La ENSANUT 2018 se encuentra dividida en cinco formularios, los cuales dan origen a nueve bases de datos con distintas poblaciones objetivo, pero todas interrelacionadas entre sí, por ejemplo, las características sobre salud y desarrollo infantil de un niño se pueden observar en dos bases de datos específicas, pero a la vez, las características del hogar en el que vive este mismo infante, características sociales o de salud de la madre, etc., se pueden apreciar en otras bases de datos distintas. Mediante el uso de algunos paquetes especializados del software $\mathrm{R}(11,12,13,14)$, se unió y filtró toda la información relacionada a los infantes de 0 a 5 años de las nueve bases de datos con el fin de obtener una sola. Finalmente se obtuvo una nueva base de 11231 individuos y 97 variables, de acuerdo a lo resumido en la $\mathrm{Ta}$ bla 1 y Tabla 2, estos individuos a su vez fueron divididos aleatoriamente en un conjunto de 7862 (70\%) para entrenamiento y 3369 (30\%) para validación, con el fin de evaluar el poder de predicción que tienen cada uno de los modelos que se usarán.

Debido a que la variable dependiente dicotómica en estudio desnutrición crónica, por su naturaleza es desbalanceada (27.97\% presentan desnutrición y $72.03 \%$ no lo hacen), se procedió a balancear las clases para el conjunto de entrenamiento usando la técnica de submuestreo, a través del paquete ROSE (15). Se analizó la cantidad de datos faltantes de cada variable, y se eliminaron aquellos casos cuyo porcentaje fue superior al 50\%, mientras que el resto de variables fueron imputadas usando el método de $\mathrm{kNN}$ vecinos más cercanos. Además, con el fin de eliminar el problema de magnitudes distintas, se centró y escaló la base de datos. Finalmente, debido a la manera en que trabajan las funciones $\mathrm{R}$ que se usó para implementar los modelos, se realizó una binarización, la cual consiste en crear tantas variables como niveles tenga una variable categórica, asignando ceros a todas las observaciones excepto a las observaciones que tienen el nivel que representa la nueva variable creada que se le asigna 1 .

\section{ANÁLISIS DE LA BASE DE DATOS.}

La primera técnica usada para determinar los factores que influyen significativamente sobre la desnutrición crónica infantil fueron los árboles de clasificación $(16,17,18,19)$. El algoritmo implementado para aplicar este modelo consistió en seleccionar aleatoriamente un subconjunto de variables, de las cuales se escoge aquellas que dividen significativamente a la muestra en cada uno de los nodos de clasificación que se van formando, minimizando la tasa de error de clasificación y asignando a cada observación la clase más común en su región del espacio de predictores. Este proceso es repetitivo y se detiene cuando aparecen categorías cuyas frecuencias respecto a la variable objetivo dejan de ser relevantes. 
Para la implementación de este algoritmo se usó el paquete rpart (20)

El problema al usar esta técnica fue que el poder predictivo del modelo resultó muy bajo, esto debido a que, en cada corrida, el algoritmo secuencial implementado siempre elegía las mismas variables con mayor frecuencia significativa; lo cual pudo provocar una omisión de variables explicativas que quizás son importantes pero que por su baja frecuencia no se tomaron en cuenta. Con el fin de solucionar este problema, se implementó un modelo más complejo, también basado en árboles, denominado Gradient Boosting (Algoritmo XGBoost) $(21,22)$, el cual va construyendo árboles de manera secuencial, donde cada nuevo árbol se crea tomando en cuenta el error de clasificación producido por las variables peor clasificadas del árbol anterior, repitiendo este proceso hasta que las potencias predictivas de todos los árboles combinados se estabilizan. Para la implementación de este algoritmo se usó el paquete xgboost.

Finalmente se implementó el modelo matemático de regresión logística binaria (familia binomial) (23), el cual por su naturaleza no presenta aleatoriedad intrínseca, lo cual hace que no se produzca el problema suscitado en el modelo de árboles. Para seleccionar las variables, se aplicó el método por pasos hacia atrás, el cual incluye en principio todas las variables explicativas del modelo para luego ir eliminando iterativamente aquellas que no aportan significativamente y se detiene cuando todos los predictores son estadísticamente significativos.

\section{RESULTADOS}

\begin{tabular}{|c|c|c|c|c|c|c|}
\hline & Descripción & B & Error estándar & valor $\mathrm{z}$ & $\operatorname{Pr}(>|z|)$ & $\exp (B)$ \\
\hline (Intercepto) & & $-0,925$ & 0,434 & $-2,132$ & $0,033^{* *}$ & 0,396 \\
\hline $\begin{array}{l}\text { FBCP1_6. } \\
\text { indígena. }\end{array}$ & $\begin{array}{l}\text { Grupo étnico } \\
\text { indígena }\end{array}$ & 0,476 & 0,089 & 5,351 & $0,000^{* * *}$ & 1,608 \\
\hline $\begin{array}{l}\text { FBCP1_7 } \\
\text { separado }\end{array}$ & $\begin{array}{l}\text { Estado civil } \\
\text { separado }\end{array}$ & 0,193 & 0,082 & 2,357 & $0,018^{* *}$ & 1,213 \\
\hline $\begin{array}{l}\text { FBCH_17 } \\
\text { no }\end{array}$ & $\begin{array}{c}\text { ¿Algún miembro del hogar tiene telf. celular? } \\
\text { no }\end{array}$ & 0,207 & 0,069 & 3,008 & $0,003^{* * *}$ & 1,229 \\
\hline FBCH_12 & Número de dormitorios de la vivienda & $-0,073$ & 0,028 & $-2,612$ & $0,009^{* * *}$ & 0,929 \\
\hline FBCP1_1 & Años cumplidos de la madre & $-0,013$ & 0,005 & $-2,388$ & $0,017^{* *}$ & 0,987 \\
\hline FBCP1_5 & Número de hijos nacidos vivos & 0,095 & 0,029 & 3,264 & $0,001^{* * *}$ & 1,099 \\
\hline $\begin{array}{l}\text { FBCP1_8 } \\
\text { Bachillerato }\end{array}$ & $\begin{array}{l}\text { Escolaridad de la madre. } \\
\text { Bachillerato. }\end{array}$ & $-0,164$ & 0,071 & $-2,302$ & $0,021^{* * *}$ & 0,848 \\
\hline $\begin{array}{l}\text { FBCP1_8 } \\
\text { Superior }\end{array}$ & $\begin{array}{l}\text { Escolaridad de la madre. } \\
\text { Superior }\end{array}$ & $-0,277$ & 0,100 & $-2,775$ & $0,006^{* * *}$ & 0,758 \\
\hline $\begin{array}{l}\text { FIPA_33 } \\
\text { No sabe - No responde }\end{array}$ & $\begin{array}{l}\text { Comió colada espesa de harina de trigo. } \\
\text { No sabe - No responde }\end{array}$ & $-0,330$ & 0,083 & $-3,981$ & $0,000^{* * *}$ & 0,719 \\
\hline FSCE_3 & Meses de embarazo cuando se hizo el primer control & 0,015 & 0,006 & 2,632 & $0,008^{\star * *}$ & 1,015 \\
\hline FSCE_12 & Primer control postparto-semanas & 0,109 & 0,038 & 2,856 & $0,004^{* * *}$ & 1,115 \\
\hline $\begin{array}{l}\text { FSCN_5 } \\
\text { más. grande }\end{array}$ & $\begin{array}{l}\text { Con respecto a otros bebes el tamaño de su hijo era: } \\
\text { Mas grande }\end{array}$ & $-0,402$ & 0,084 & $-4,761$ & $0,000^{* * *}$ & 0,668 \\
\hline $\begin{array}{l}\text { FSCN_5 } \\
\text { pequeño }\end{array}$ & $\begin{array}{c}\text { Con respecto a otros bebes el tamaño de su hijo era: } \\
\text { Mas pequeño }\end{array}$ & 0,271 & 0,086 & 3,148 & $0,002^{* * *}$ & 1,311 \\
\hline $\begin{array}{l}\text { FSCCN_21 } \\
19-23\end{array}$ & $\begin{array}{l}\text { Grupo de edad } \\
\quad 19-23\end{array}$ & 0,430 & 0,090 & 4,757 & $0,000^{* * *}$ & 1,537 \\
\hline $\begin{array}{l}\text { FSCCN_21 } \\
31-35\end{array}$ & $\begin{array}{l}\text { Grupo de edad } \\
\quad 31-35\end{array}$ & 0,339 & 0,093 & 3,646 & $0,000^{* * *}$ & 1,404 \\
\hline $\begin{array}{l}\text { FIEI_1 } \\
\text { si }\end{array}$ & $\begin{array}{l}\text { ¿Ha tenido diarrea en las últimas dos semanas? } \\
\qquad \mathrm{Si}\end{array}$ & 0,842 & 0,416 & 2,024 & $0,043^{* *}$ & 2,321 \\
\hline
\end{tabular}

Tabla 3. Resultados de las variables significativas en el modelo de regresión logística.

Fuente: Elaboración Propia.

En el caso del modelo de regresión, se comprobó mediante el omnibus test, que el modelo planteado muestra una relación significativa entre las variables independientes y la dependiente ( $\mathrm{p}$ valor del estadístico chi cuadrado igual a $1.43 \times 10$ $\left.{ }^{45}\right)$. La Tabla 3 muestra los coeficientes signifi- 
cativos del modelo, su error estándar, valor Z, p valor y probabilidad asociada de ocurrencia con la variable dependiente (desnutrición crónica). Respecto a los $\mathrm{p}$ valores que indican si un coe- ficiente es o no significativo, se ha colocado ** indicando que se cumple a un nivel de significancia del $5 \% y^{* * *}$ cuando lo hace a una significancia del $1 \%$.

\begin{tabular}{|l|l|l|l|l|l|l|l|}
\hline \multicolumn{2}{|c|}{} & \multicolumn{6}{l}{ Presenta Desnutrición crónica } \\
\cline { 2 - 8 } & \multicolumn{2}{|l}{ Modelo de Regresión } & \multicolumn{2}{l|}{ Modelo de árboles } & \multicolumn{2}{l|}{ Modelo Gradeint Boosting } \\
\cline { 3 - 8 } & & No & Si & No & Si & No & Si \\
\hline \multirow{2}{*}{ Presenta Desnutrición crónica } & No & 1774 & 719 & 1546 & 465 & 1687 & 510 \\
\cline { 2 - 8 } & $\mathrm{Si}$ & 473 & 403 & 888 & 470 & 747 & 425 \\
\hline
\end{tabular}

Tabla 4. Matriz de confusión para los modelos de regresión, árboles y gradeint boosting. Fuente: Elaboración Propia.

Al comprobar el poder predictivo de este modelo, usando la muestra de entrenamiento, se observó en la matriz de confusión que 2177 de los 3369 individuos fueron clasificados correctamente, la Tabla 4. por lo que el poder predictivo de este modelo se establece en $64.6 \%$.

La matriz de confusión completa se presenta en

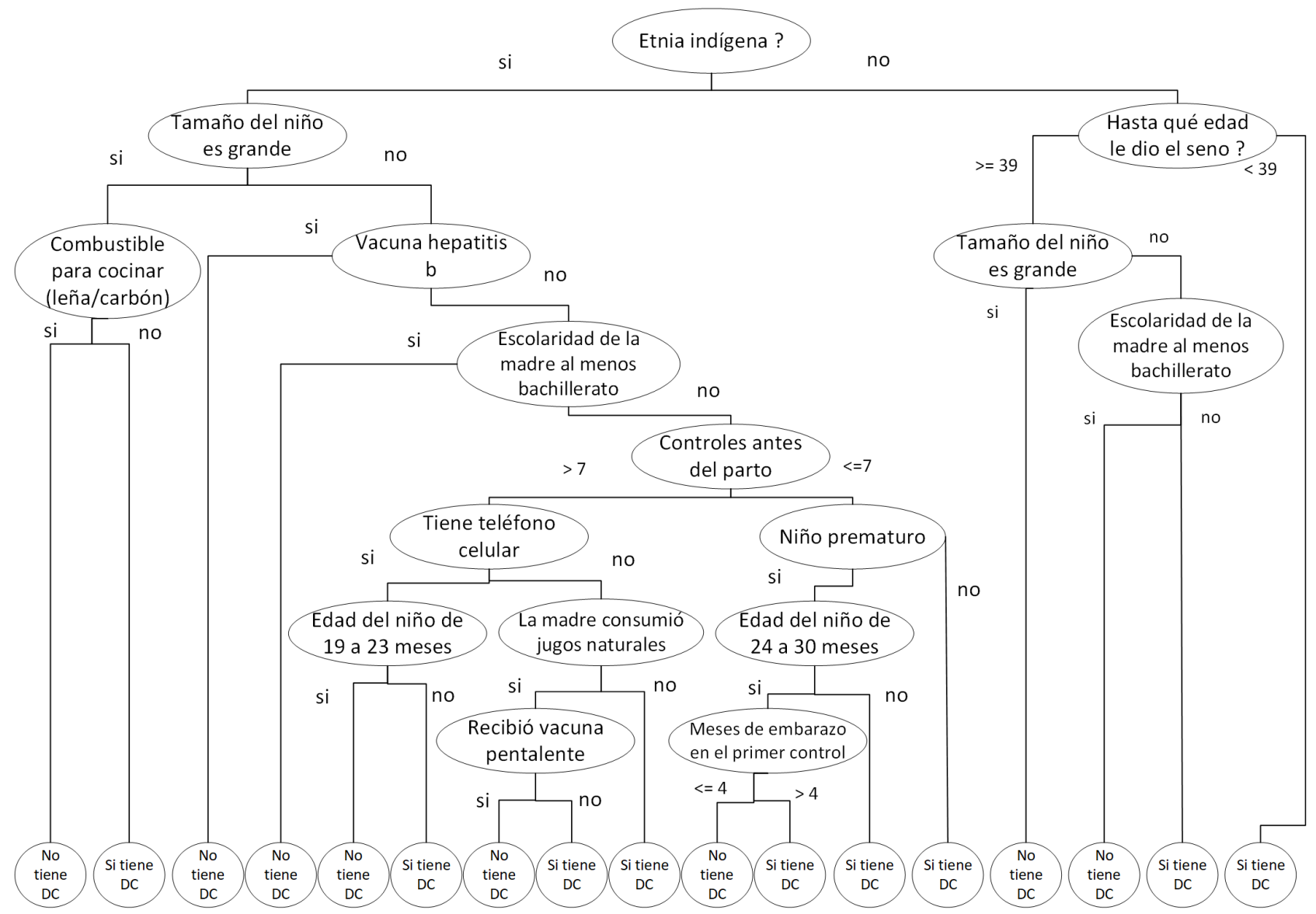

Figura 2. Modelo de árbol de decisión para predecir la desnutrición crónica infantil Fuente: Elaboración Propia.

Respecto al modelo de árboles de clasificación, la Figura 2 muestra que se forman 15 nodos intermedios, los cuales representan las variables o factores de mayor influencia. La variable más significativa o influyente en la desnutrición crónica de acuerdo a este modelo (nodo raíz) pertenece a la categoría indígena de la variable grupo étnico (FBCP1_6). En adelante, se muestran las variables en orden de influencia, vistas en la figura desde arriba hacia abajo. De acuerdo a la matriz de confusión, 2016 de los 3369 individuos considerados en la muestra de evaluación fueron clasificados correctamente, lo cual nos da un poder predictivo del 59.8\% (la matriz de confusión completa se presenta en la Tabla 4.). Sin embargo, como ya habíamos mencionado, con la finalidad de mejorar este indicador, se implementó el algoritmo del modelo Gradient Boosting, el cual presentó un poder predictivo de $62.7 \%$ (la matriz de confusión completa se presenta en la Tabla 4.), lo 
cual, a nuestro criterio no representa una mejora significativa como teóricamente se suponía, por lo que podemos conluir que la capacidad de ajuste es estable en el árbol presentado en la Figura 2 y se puede tomar decisiones a partir de ello.

\section{DISCUSIÓN}

\section{FACTORES ASOCIADOS A LA DESNUTRI- CIÓN CRÓNICA EN EL ECUADOR.}

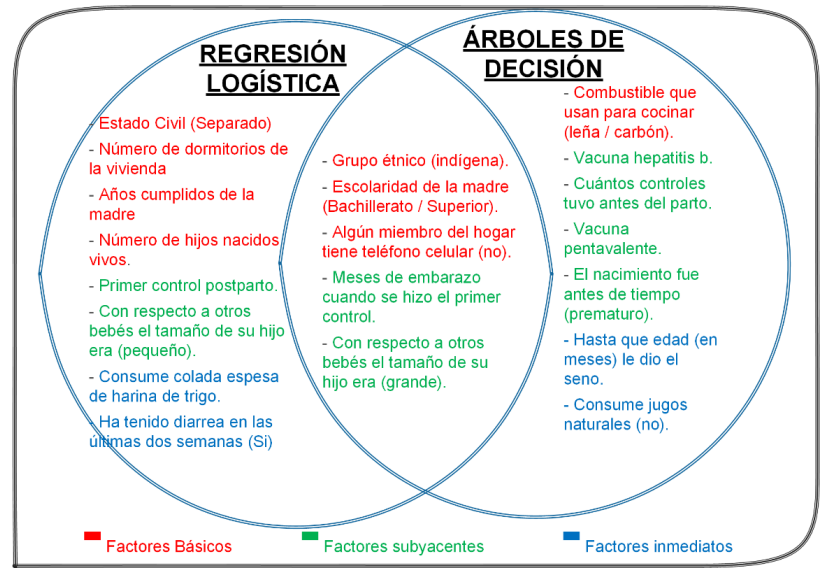

Figura 3. Factores asociados a la desnutrición crónica infantil de acuerdo a los modelos de regresión logística y árboles de decisión. Intersección entre ellos.

Fuente: Elaboración Propia.

La Figura 3 muestra un resumen de los factores que fueron considerados como significativos en cada uno de los modelos (tomando en cuenta para el caso de regresión un nivel de significancia del 5\%), además de aquellos que fueron comunes, diferenciándolos por el tipo al que pertenecen de acuerdo con el marco teórico de clasificación propuesto por la UNICEF (10). Es importante recalcar en este punto que, de acuerdo a esta referencia teórica, si se mejoran las condiciones de las variables que pertenecen a los factores básicos (color rojo), esto incidirá directamente y de manera positiva en los factores subyacentes e inmediatos (color verde y azul respectivamente), razón por la cual, es evidente que debe existir atención principalmente en la creación de condiciones necesarias para mejorar las características de estos factores básicos.

Si analizamos los resultados de manera más detallada, el modelo de regresión logística (Tabla 3), muestra que la categoría indígena, perteneciente a la variable grupo étnico, presenta una influencia sobre la desnutrición crónica infantil por encima de las otras variables independientes, de esta manera, si el niño es indígena tiene $60.8 \%$ mayor probabilidad de tener desnutrición crónica ante niños de otros grupos étnicos (FBCP1_6). Seguida de esta variable, en orden de significancia encontramos que si el niño resulta ser más pequeño en relación a otros niños de su misma edad (FSCN_5), la probabilidad de que el niño tenga desnutrición crónica aumenta en $31.1 \%$. Si algún miembro del hogar no tiene comunicación mediante un teléfono celular (FBCH_17), el niño tiene $22.9 \%$ mayor probabilidad de tener desnutrición crónica. Si el estado civil (FBCP1_7) de los padres es separado, la probabilidad de que el niño tenga desnutrición aumenta en $21.3 \%$. Si el número de hijos nacidos vivos en el hogar (FBCP1_5) aumenta, también lo hace la probabilidad de desnutrición en $9.9 \%$. Si la madre se tarda en realizar su primer control de embarazo (FSCE_3), la probabilidad de que el niño tenga desnutrición crónica aumenta en $1.5 \%$ y si se tarda en su primer control postparto (FSCE_12) esta probabilidad aumenta en $11.5 \%$. Además de estas, encontramos las siguientes relaciones negativas: Si el niño es más grande con respecto a otros bebes de su misma edad (FSCN_5.) la probabilidad de que el niño tenga desnutrición crónica disminuye en $33.2 \%$. Si la madre tiene un nivel de escolaridad superior (FBCP1_8.) la probabilidad de que el niño tenga desnutrición crónica disminuye en $24.2 \%$, mientras que si tiene un nivel de educación media - bachillerato, la probabilidad disminuye en $15.2 \%$ y finalmente a medida que el número de dormitorios que posee la vivienda en la que vive el niño crece (FBCH_12), la probabilidad de tener desnutrición crónica disminuye en un $7.1 \%$.

Por otra parte, los modelos basados en árboles (Figura 2) revelan que la variable más influyente para que un niño tenga desnutrición sigue siendo el grupo étnico en la categoría indígena, seguido en orden de importancia de: La edad hasta que fue amamantado el infante (FIPA_2), el tamaño del bebe respecto a otros (FSCN_5), la escolaridad de la madre (FBCP1_8), el combustible que se usa en casa para cocinar (FBCH_14), la dosis de vacuna contra la hepatitis (FSCCN_9), el número de controles antes del parto (FSCE_4), la tenencia de un teléfono celular de un miembro del hogar (FBCH_17), la alimentación de la ma- 
dre con frutas naturales (FIPA_19), la dosis de la vacuna pentavalente (FSCCN_12), el nacimiento antes de tiempo (FSCE_9) y primer control del embarazo (FSCE_3).

\section{ANÁLISIS COMPARATIVO CON LA REALI- DAD DE OTROS PAÍSES.}

Aunque el estudio sobre factores asociados a la desnutrición crónica infantil en países que viven realidades cercanas a la nuestra es escaso, los estudios realizados en la región y en países en vías de desarrollo muestran escenarios similares a los encontrados en la presente investigación para nuestro país.

En Perú, se conoce que los factores básicos de mayor influencia son: Bajos niveles de escolaridad de los padres, residir en el área rural y mala calidad de la vivienda (piso y servicios higiénicos). Respecto a la escolaridad, si la madre tiene instrucción primaria, un niño tiene hasta $61 \%$ más probabilidad de tener desnutrición, lo cual resulta análogo a la realidad encontrada en la presente investigación para Ecuador, donde, si la madre tiene un alto grado de escolaridad, la probabilidad de que el niño presente desnutrición disminuye en $33.2 \%$. Respecto a residir en el área rural (población en su mayoría indígena), en Perú es $57.9 \%$ más probable que estos niños tengan desnutrición comparados con los que viven en la zona urbana, lo cual parece ser similar a lo que se encontró en nuestro país, específicamente con la etnia indígena, los cuales como ya se mencionó son $60.8 \%$ más propensos a tener desnutrición crónica. Respecto a la calidad de la vivienda, se sabe que en Perú el riesgo asciende a $57 \%$ más en niños que viven en casas de mala calidad, lo cual se podría comparar con la variable número de dormitorios, así, en Ecuador, mientras más habitaciones exista, el riesgo disminuye. Respecto a los factores subyacentes, en Perú, no tener un parto institucionalizado, tener más de 4 hijos, bajo peso al nacer y no tener una inmunización completa aumenta la probabilidad de desnutrición en 19\%, 1.8\%, 21.4\% y $17.6 \%$ respectivamente, mientras que en Ecuador variables similares como: Primer Control Post parto, tamaño del bebe, nacimiento prematuro y vacunas (hepatitis B y pentavalentes), también resultaron significativas en la desnutrición crónica. Final- mente, respecto a los factores inmediatos, en Perú, la duración de la lactancia, la alimentación adecuada y presentar alguna afectación de salud (diarrea, fiebre, tos) son factores que aumentan significativamente la probabilidad de desnutrición, lo cual es similar a la realidad de Ecuador, donde se determina que las variables: Consume colada espesa, Ha tenido diarrea, Hasta qué edad le dio el seno y consume jugos naturales, afectan de igual manera a la probabilidad de desnutrición crónica $(24,25)$.

En Brasil y Haití, aunque el proceso de muestreo no se basa en el marco teórico de la UNICEF y solo se hace el estudio para cierto grupo de individuos en específico (Municipios del estado de Acre y niños indígenas de Mangueirinha, Paraná para el caso de Brasil y niños de 6 a 24 meses para el caso de Haití), los resultados también son similares a los encontrados en nuestro país. De esta manera, en Brasil, principalmente la condición económica de los padres, su escolaridad y su edad, el número de hijos, la ausencia de la madre, el no acceso al servicio de alcantarillado y electricidad fueron factores determinantes $(26,27)$, los cuales tienen mucha similitud con las variables: Escolaridad de la madre, años cumplidos de la madre, número de hijos nacidos vivos, estado Civil de los padres y número de dormitorios de la vivienda, las cuales fueron significativas en nuestro país y se observa que todas ellas están clasificadas dentro de los factores básicos, lo cual da a entender que en Brasil se realizó el estudio poniendo énfasis particularmente en este tipo de factores, los cuales si bien es cierto son las más importantes pero a nuestro parecer no nos deja conocer la realidad de la situación a detalle.

En Haití, la escolaridad de la madre, su acceso a los medios de comunicación, el índice de riqueza del hogar, el espaciamiento entre los nacimientos, el número de hijos, el estado nutricional de la madre, el número de consulta prenatales durante el embarazo y la defecación al aire libre a nivel de comunidad son los principales factores que influyen en la desnutrición crónica infantil (28), sin duda, estos aspectos son muy similares a las variables medidas en nuestro país por medio de la ENSANUT: Escolaridad de la madre, algún miembro del hogar tiene teléfono celular, número de hijos nacidos vivos y controles postparto, 
los cuales están en las categorías de factores básicos y subyacentes.

Es importante indicar que para estos dos últimos países (Brasil y Haití), se usaron metodologías distintas a las usadas en la presente investigación, como por ejemplo análisis de frecuencias e inferencias basadas en ellas, o regresiones lineales y métodos de descomposición (por ejemplo en el caso de Haiti, con variables cuantitativas obtenidas a partir de las encuestas de mortalidad y morbilidad), los cuales son métodos que si bien es cierto nos permiten conocer si una variable independiente es o no significativa para explicar una dependiente (desnutrición crónica), estos no nos dejan conocer una medida de la probabilidad de ocurrencia o influencia de la variable independiente sobre la dependiente, como sí lo hace la metodología de regresión logística usada en la presente investigación.

\section{CONCLUSIONES}

De acuerdo a los resultados obtenidos en este trabajo, en el Ecuador, si un niño es indígena, la escolaridad de la madre es inferior al bachillerato, algún miembro del hogar no tiene teléfono celular, la madre demora en realizarse el primer control de embarazo, el tamaño del infante no es el adecuado, sus padres son separados, existen menos dormitorios en la vivienda, es mayor el número de hijos nacidos vivos en el hogar, el combustible que usan para cocinar es leña o carbón, la madre se tarda en realizarse el primer control postparto, el niño es prematuro, la madre se hizo pocos controles antes del parto, al niño le faltan las vacunas de la hepatitis b y pentavalente, el niño no consume colada espesa de harina de trigo, no ha tenido diarrea durante los las últimas semanas, no consume jugos naturales y su madre no le dio el seno durante largo tiempo, entonces son muy altas las posibilidades de que un niño presente desnutrición crónica, lo cual como ya se mencionó afecta no solo el estado del infante en sus primeros años, sino durante toda su vida.

Como ya se había mencionado: La desnutrición crónica constituye un problema de salud pública y social, la cual aumenta el riesgo de morbilidad y mortalidad, además afecta negativamente en el crecimiento y desarrollo del infante, acarreando impactos negativos que se extienden hasta la edad adulta, la cual reduce la capacidad de trabajo, afectando a la productividad económica de un país. En este contexto, y luego de medir el nivel de afectación que tienen las variables estudiadas sobre la desnutrición crónica infantil, creemos que las políticas en nuestro país deben enfocarse principalmente en aquellas variables o factores que de acuerdo al presente estudio presentan una influencia significativamente más alta, recordando a la vez que de acuerdo al marco teórico de la UNICEF, si mejoramos las condiciones principalmente en las variables pertenecientes a los factores básicos, también lo harán de manera indirecta las variables pertenecientes a los factores subyacentes e inmediatos. Creemos que tomado en cuenta estas consideraciones técnicas, los recursos que son destinados a erradicar la desnutrición se optimizarán, pero sobre todo, creemos que el problema disminuirá significativamente.

$\mathrm{R}$ eferencias

1. UNICEF. Estado mundial de la infancia 2008 - Resumen Ejecutivo: Supervivencia Infantil.: Unicef; 2007.

2. INEC. Boletín Técnico: Encuesta Nacional de Salud y Nutrición 2018. Quito - Ecuador.; 2019.

3. SENPLADES. Plan Nacional de Desarrollo 2017-2021. Toda una Vida Quito - Ecuador; 2017. 4. INEC. Encuesta Nacional de Salud y Nutrición ENSANUT. [Online]. [cited 2021 Enero. Available from: https://www.ecuadorencifras.gob.ec/salud-salud-reproductiva-y-nutricion/.

5. Trujillo M. Factores asociados a desnutrición crónica infantil en niños menores de cinco años de edad en el Perú: Sub-análisis de la Endes 2018. Universidad Ricardo Palma. 2020; 1(1).

6. Pérez J, Echavarría F. Árboles de clasificación vs regresión logística en el desarrollo de competencias genéricas en ingeniería. Computación y Sistemas. 2018; 22(4).

7. Demir E. A decision support tool for predicting patients at risk of readmission: A comparison 
of classification trees, logistic regression, generalized additive models, and multivariate adaptive regression splines. Decision Sciences. 2014; 45(5).

8. Charris L. Análisis comparativo de los algoritmos de árboles de decisión en el procesamiento de datos biológicos. Investigación y desarrollo en TIC. 2018; 9(1).

9. Andrade V, Flores P. Comparativa entre classification trees, random forest y gradient boosting; en la predicción de la satisfacción laboral en Ecuador. Ciencia Digital. 2018 Diciembre; 2(4).

10. UNICEF. Estado mundial de la infancia - Nutrición. New York: Fondo de las Naciones Unidas para la Infancia; 1998.

11. R CT. R: A Language and Environment for Statistical Computing. Vienna - Austria: R Foundation for Statistical Computing; 2020.

12. Kuhn M. caret: Classification and Regression Training. 2020. R package version 6.0-86.

13. Therneau T, Atkinson B. rpart: Recursive Partitioning and Regression Trees. 2019. R package version 4.1-15.

14. Chen T, He T, Benesty M, Khotilovich V, Tang Y, Cho H, et al. xgboost: Extreme Gradient Boosting. 2021. R package version 1.3.2.1.

15. Lunardon N, Giovanna M, Nicola T. ROSE: a Package for Binary Imbalanced Learning. R Journal. 2014; 6(1).

16. Maimon O, Rokach L. Data mining with decision trees: theory and applications: World scientific; 2014.

17. Breiman L. Classification and regression trees.: Wadsworth International Group; 1984.

18. Buntine W. Learning classification trees. Statistics and computing. 1992; 2(2).

19. Rokach L, Oded M. Classification trees. In Data mining and knowledge discovery handbook Boston: Springer; 2009.

20. Therneau T, Atkinson B. rpart: Recursive Partitioning and Regression Trees. 2019. R package version 4.1-15.

21. Hastie T, Tibshirani R, Friedman J. Boosting and additive trees. In The elements of statistical learning New York: Springer; 2009.

22. Elith J, hastie T. A working guide to boosted regression trees. Journal of Animal Ecology. $2008 ; 77(4)$.

23. tolles J, Meurer W. Logistic regression: relating patient characteristics to outcomes. Jama. 2016; 316(5).

24. Arocena V. Factores asociados a la Desnutrición Crónica Infantil en el Perú, 1996-2007. Instituto Nacional de Estadística e Informática. 2009 octubre.

25. Arocena V. Factores asociados a la desnutrición crónica infantil en Perú: una aplicación de modelos multinivel. Revista Latinoamericana de Población. 2010; 4(6).

26. Kühl A, Corso A, Leite M, Bastos J. Perfil nutricional e fatores associados à ocorrência de desnutrição entre crianças indígenas Kaingáng da Terra Indígena de Mangueirinha, Paraná, Brasil. Cadernos de Saúde Pública. 2009; 25.

27. Souza O, Benicio M, Castro T, Muniz P, Cardoso M. Desnutrição em crianças menores de 60 meses em dois municípios no Estado do Acre: prevalência e fatores associados. Revista brasileira de Epidemiologia. 2012; 15(1).

28. Généus W, Lachaud, Gayet I. Factores asociados al descenso de la desnutrición crónica de los niños de 6 a 24 meses en Haití en el período 1994-2017. Notas de Población. 2020. 